\title{
Estado Periodontal y Necesidad de Tratamiento en Pacientes GES 60 Años de Villa Alemana
}

\author{
Periodontal Status and Treatment Needs in 60 Years-old \\ GES Patients from Villa Alemana
}

\author{
Rojas González C ${ }^{1}$, Segovia Chamorro J22, Raccoursier Schwerter V³, Godoy Olave J4, Lopetegui Buschmann MS ${ }^{5}$
}

\section{RESUMEN}

Introducción: El envejecimiento es un proceso fisiológico deteriorativo que se observa desde la sexta década de la vida. Si bien los cambios demográficos de Chile revelan un envejecimiento poblacional, no existe suficiente información sobre el estado periodontal de los adultos de 60 años en nuestro país. Actualmente el Programa GES "Salud Oral Integral para el Adulto de 60 Años" tiene como objetivo mejorar su calidad de vida. Objetivo: Determinar el estado periodontal de los adultos de 60 años pertenecientes a los consultorios municipalizados de Villa Alemana y su necesidad de tratamiento. Pacientes y Método: Se examinaron a 124 pacientes voluntarios, seleccionados de forma aleatoria simple. Se les realizó un examen registrándose: higiene oral, índice hemorrágico, pérdida de inserción clínica, profundidad de sondaje y PSR. Se realizó el análisis estadístico y test de Chi-cuadrado. Resultados: El $90.32 \%$ presentó una mala higiene oral, en promedio presentaron un índice hemorrágico de $68.42 \%$, el $100 \%$ presentó pérdida de inserción clínica y el $14.51 \%$ presentó en promedio profundidades de sondaje mayores o iguales a $5 \mathrm{~mm}$. La totalidad de los adultos de 60 años necesita algún tipo de tratamiento periodontal, siendo un $82.3 \%$ el que necesita un tratamiento periodontal complejo. Conclusiones: Los pacientes GES de 60 años presentaron un mal estado periodontal determinado por higiene oral, índice hemorrágico, pérdida de inserción clínica y profundidad de sondaje, mostrando un importante deterioro de la salud periodontal de dicha población. La totalidad de los adultos de 60 años necesita tratamiento periodontal de algún tipo, siendo un $82.3 \%$ el que necesita tratamiento periodontal complejo.

Rev. Clin. Periodoncia Implantol. Rehabil. Oral Vol. 3(2); 86-89, 2010.

Palabras clave: Periodontitis, epidemiología, adulto mayor, GES 60 años.

\begin{abstract}
Introduction: Aging is a physiological and deteriorative process that begins in the sixth decade of life. Demographic changes in Chile reveal an aging population. In our country there is a lack of information about the periodontal status of the group of adults of 60 years-old. Nowadays, the GES Program "Integral Oral Health for 60 Years-old Adults" aims to improve their quality life. Objectives: Determine the periodontal status and treatment needs of adults aged 60 belonging to primary care health center of Villa Alemana. Patients and Methods: A total of 124 patients, randomly selected, were examined. It was effectuated an extra and intraoral examination, registering: oral hygiene, bleeding index, clinical attachment loss, probing depth and PSR. Statistical analysis and Chi-square test were done. Results: $90.32 \%$ of sample showed a poor oral hygiene, the patients presented a bleeding index of $68.42 \%$ on average, $100 \%$ of sample presented clinical attachment loss and $14.51 \%$ showed on average probing depths greater than or equal to $5 \mathrm{~mm}$. All the patients need some periodontal treatment, and an $82.3 \%$ needs comprehensive periodontal treatment. Conclusions: 60 years-old adults showed a poor periodontal status determined by oral hygiene, bleeding index, clinical attachment loss and probing depths, showing an important deterioration of periodontal health of this population. All the 60 years-old adults need some kind of periodontal therapy, while an $82.3 \%$ needs a comprehensive periodontal treatment.
\end{abstract}

Rev. Clin. Periodoncia Implantol. Rehabil. Oral Vol. 3(2); 86-89, 2010.

Key words: Periodontitis, epidemiology, older adults, 60 years old GES.

\section{INTRODUCCIÓN}

El envejecimiento es un proceso fisiológico deteriorativo asociado a una disminución de la viabilidad y un aumento de la vulnerabilidad, manifestado en un incremento de la probabilidad de morir de acuerdo con el aumento de la edad cronológica. Este proceso comienza a dar evidencias a partir de la sexta década de la vida(1).

Chile está viviendo una etapa avanzada de transición al envejecimiento demográfico de su población ${ }^{(2)}$. Según los datos del último Censo, se proyecta que para el año 2025 la población de adultos mayores alcanzará un $16 \%$, con un total de 3.000 .000 de personas mayores de 60 años $^{(1)}$. Debido a este rápido incremento es que la organización y atención en salud -para este grupo poblacional- toma cada vez más relevancia(3).

Los adultos mayores son el grupo con mayor morbilidad bucal, por no haber recibido durante su vida suficientes medidas de prevención o tratamientos adecuados y oportunos para recuperar su salud bucal(4). El periodonto en los adultos mayores refleja los cambios relacionados con la edad y la acumulación de experiencia de enfermedad y traumas en el tiempo, demostrándose que la edad produce ciertos cambios fisiológicos en el periodonto, aunque estos cambios por sí solos no son responsables de la destrucción periodontal(5). A su vez influyen adversamente muchos factores como son las enfermedades crónicas, factores bucales y medicamentos ${ }^{(6)}$.

El Gobierno de Chile en el año 2006 puso en marcha el programa GES (Garantías Explicitas en Salud) "Salud Oral Integral para el Adulto de 60 Años", cuyo objetivo es que el adulto de 60 años obtenga una Salud Oral Integral que lo acompañe durante el nuevo período de vida que comienza ${ }^{(4)}$, a través de un tratamiento que incluye la terapia periodontal.

1. Cirujano Dentista. Docente Ad-Honorem, Cátedra Periodoncia. Facultad de Odontología, Universidad de Valparaíso. Chile.

2. Alumno. Facultad de Odontología, Universidad de Valparaíso. Chile.

3. Cirujano Dentista. Chile.

4. Especialista en Periodoncia. Docente Cátedra Periodoncia. Facultad de Odontología, Universidad de Valparaíso. Chile.

5. Especialista en Periodoncia. Docente Cátedra Periodoncia. Facultad de Odontología, Universidad de Valparaíso. Chile.

Correspondencia autor: Carla Rojas G. carlarojasg@yahoo.com. Subida Carvallo 211, Playa Ancha, Valparaíso. Chile. Trabajo recibido el 09/06/2010. Aprobado para su publicación el 29/08/2010. 
Villa Alemana es una de las comunas con mayor porcentaje de adultos mayores en la Quinta Región $(13.1 \%)^{(7)}$, por lo que fue seleccionada como objetivo de nuestro estudio, dada la susceptibilidad de dicha población a padecer enfermedad periodontal.

\section{PACIENTES Y MÉTODOS}

La presente investigación corresponde a un estudio descriptivo transversal, el cual determinó el estado periodontal y la necesidad de tratamiento de los adultos beneficiarios del programa GES "Salud Oral Integral para el Adulto de 60 Años" pertenecientes a los consultorios municipalizados de Villa Alemana, bajo una metodología estandarizada.

Se obtuvo una muestra aleatoria por conglomerado a partir de las bases de datos disponibles en cada consultorio, independiente de su sexo, en relación a la población total inscrita en éstos, para seleccionar al azar la muestra mediante un muestreo aleatorio simple con ayuda de una tabla de número aleatorios. Vía telefónica se citó a los pacientes para que acudieran al examen odontológico. En forma previa al inicio del examen se les informó a los pacientes sobre los objetivos del estudio, explicándoles además, la necesidad de firmar un consentimiento informado, a través del cual aprobaron su participación en la presente investigación.

La muestra consistió en 124 pacientes de 60 años, hombres o mujeres, que presentaron en la boca al menos tres dientes, que acudieron voluntariamente. El tamaño de la muestra se calculó con la fórmula $n=z^{2} p(1-p) / d^{2}$, con un nivel de confianza del $95 \%$, una prevalencia muestral del $97 \%^{(8)}$ y un error muestral del $3 \%$. La unidad de muestra seleccionada fueron los pacientes GES de 60 años.

Los datos fueron recogidos a través de una ficha clínica diseñada especialmente para el registro de los datos de este estudio. Ésta se dividió en dos partes: Anamnesis y Examen Odontológico. La anamnesis consistió en una entrevista sobre datos generales del paciente y fue realizada por un encuestador perteneciente al grupo de investigación, guiándose por el instructivo de llenado de ficha.

El examen odontológico fue efectuado bajo condiciones estandarizadas en las dependencias establecidas por cada consultorio. Este examen consistió en un examen dental y periodontal. El examen de los pacientes fue efectuado por dos examinadores, pertenecientes al grupo de investigación, se repasaron y especificaron las definiciones operacionales de las variables a medir, además de clarificar cualquier diferencia de concepto que pudiese existir. Se repasó también el modo de llenado de la ficha de registro de información, los instrumentos utilizados para el examen y las condiciones estandarizadas en las que éste se realizó. Luego se procedió a la calibración propiamente tal, tanto intraexaminador como interexaminador.

El examen de los pacientes que participaron en este estudio se realizó con luz directa de la lámpara del equipo, instrumental de examen básico (sonda de caries curva, espejo $\mathrm{n}^{\circ} 5$ y pinza de curación), sonda Williams Goldman-Fox (American Eagle) para medir la profundidad de sondaje y el nivel de inserción clínico y sonda Nabers 2N (American Eagle) para determinar la existencia de compromiso de furca.

En el examen intraoral se evaluaron los siguientes parámetros: presencia de caries, cálculo, profundidad de sondaje, nivel de inserción clínica, movilidad, compromiso de furca, índice Hemorrágico (índice Gingival Simplificado de Lindhe), índice de Higiene Oral de O'Leary y PSR (Periodontal Screening and Recording). El parámetro de índice de Higiene Oral fue el último que se evaluó en cada examen, entregando al paciente una tableta reveladora (Sanora ${ }^{\circledR}$, Biotoscana S.A.), la cual era disuelta en su boca durante 1 minuto para posteriormente enjuagarse $y$ ser evaluado por el examinador.

Para efectos del análisis estadístico se tomaron en cuenta los datos de la muestra general y además se separó en subgrupos. En algunos casos se calcularon los intervalos de confianza, las medias, la desviación estándar, el mínimo, el máximo y la moda. En los casos necesarios se calculó el nivel de significancia, con un nivel de confianza del $95 \%$ a través del test de Chi-cuadrado $\left(X^{2}\right)$ de Pearson. La base de datos se confeccionó utilizando el software Microsoft Office Excel 2007 y los datos fueron tabulados y codificados para ser transferidos al software SPSS (Statistical Package for the Social Sciences) versión 16.0 para su análisis estadístico. Este estudio fue previamente aprobado por el Comité de Ética de la Facultad de Odontología de la Universidad de Valparaíso.

\section{RESULTADOS}

Del total de los adultos de 60 años examinados, 84 correspondieron a mujeres $(67.74 \%)$ y 40 a hombres $(32.26 \%)$. Se examinaron 2.054 dientes en total, con un promedio de dientes en boca de 16.56. La moda fue igual a 25 dientes, mientras que la máxima fue de 29 .

\section{Higiene Oral}

Los resultados del índice de Higiene Oral de O'Leary se observan en la Figura 1, dividiéndose por mala higiene (0-50\% con sitios libres de biofilm), regular higiene (sitios libres de biofilm de 51 a un 79\%) y buena higiene (mayor a $80 \%$ de los sitios libres de biofilm). El promedio de higiene fue de un $22.3 \%$ siendo un resultado malo. El mejor índice de higiene fue de un $82.3 \%$ siendo el único paciente que presentó una buena higiene oral, mientras el peor fue de $0 \%$. Cabe mencionar que la moda para higiene oral fue de $0 \%$, con 25 pacientes con ese valor, correspondiente al $20.1 \%$ del total de los adultos de 60 años.

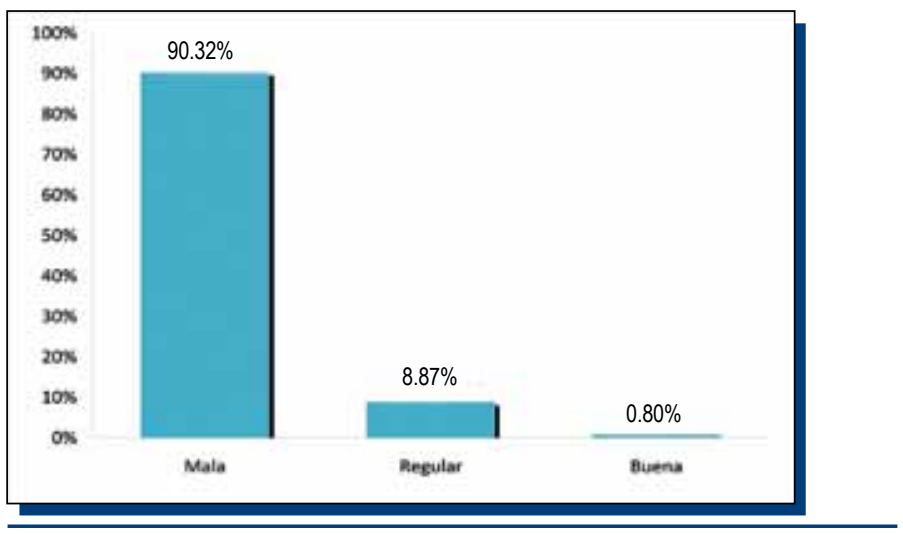

Figura 1. Porcentaje de higiene oral en los adultos de 60 años.

\section{2. Índice Hemorrágico}

Respecto al índice Hemorrágico, la muestra presentó un promedio de $68.42 \%$ de sitios hemorrágicos al sondaje, siendo $6.3 \%$ la mínima y $100 \%$ la máxima. La moda fue de $100 \%$, con 25 pacientes en ese valor, correspondientes al $20.1 \%$.

\section{Profundidad de Sondaje}

Las profundidades de sondaje promedio por paciente se pueden observar en la Figura 2. Cabe mencionar que del total de 2.054 dientes examinados, 505 dientes presentaron profundidad de sondaje $\geq 5 \mathrm{~mm}$, correspondientes a un $24.6 \%$ del total. El paciente que tuvo más dientes con profundidad de sondaje $\geq 5 \mathrm{~mm}$, correspondió a 20 dientes afectados. También se encontraron 33 pacientes $(26 \%)$ que no presentaron ningún diente con profundidad de sondaje $\geq 5 \mathrm{~mm}$. Los otros 91 pacientes presentaron al menos un diente con profundidad de sondaje $\geq 5 \mathrm{~mm}(73.9 \%)$.

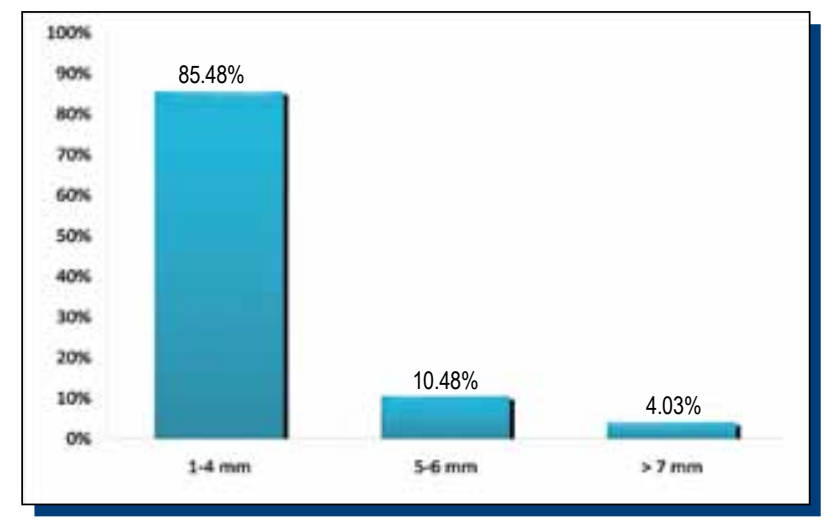

Figura 2. Porcentaje de adultos de 60 años, según intervalo de profundidad de sondaje. 


\section{Nivel de Inserción Clínica}

El total de los pacientes presentaron pérdida de inserción clínica. Se observan los valores promedios de NIC por paciente en la Figura 3. Cabe mencionar que el $100 \%$ de los dientes de los pacientes presentaron pérdida de inserción clínica de al menos $1 \mathrm{~mm}$.

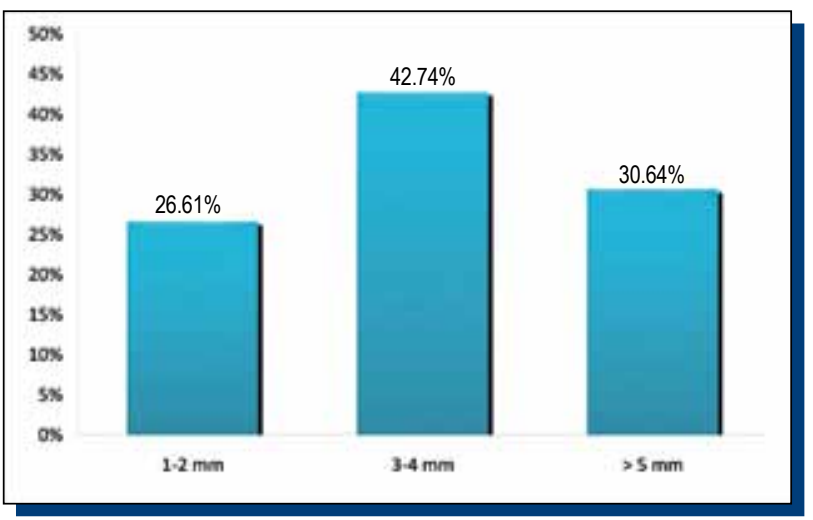

Figura 3. Porcentaje de adultos de 60 años, según intervalo de nivel de inserción clínica.

\section{PSR (Periodontal Screening and Recording)}

Los resultados del PSR se pueden observar en la Figura 4. Se determinó que la totalidad de los adultos de 60 años necesita tratamiento periodontal de algún tipo, siendo un $82.3 \%$ el que necesita tratamiento periodontal complejo (correspondiente a los códigos 3 y 4): confección de periodontograma, radiografías, desbridamiento y pulido y alisado radicular donde corresponda. Mientras que un 32.3\% necesita derivación al especialista por código 4, al tener profundidades muy aumentadas.

El código asterisco $\left(^{*}\right)$ corresponde a lesiones que deben ser tratadas por el periodoncista, independiente del código numérico del PSR, las cuales corresponden a presencia de recesiones mayores a $3.5 \mathrm{~mm}$, alteraciones mucogingivales, compromiso de furca y movilidad dentaria. En este caso correspondió al $70.2 \%$ de los pacientes examinados.

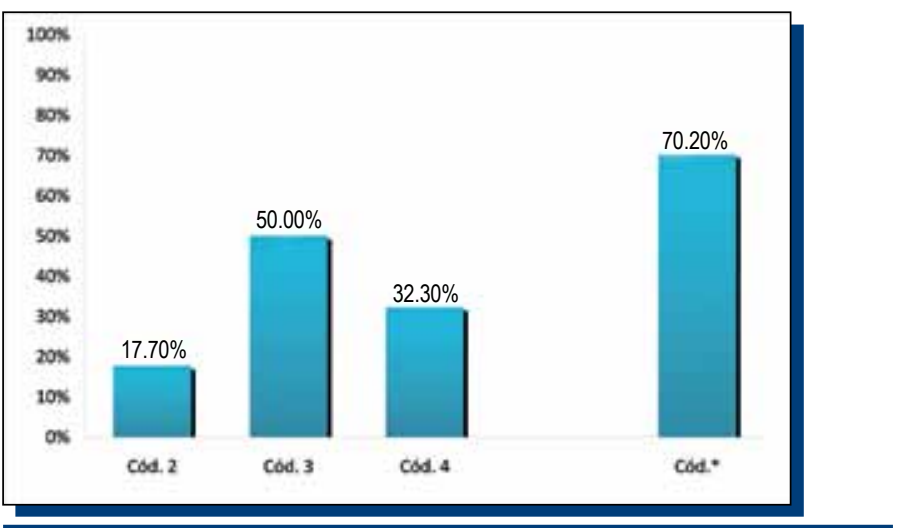

Figura 4. Distribución de los adultos de 60 años, según código PSR.

\section{Otros Resultados}

De los 124 adultos de 60 años, el $27.4 \%$ presentó movilidad dentaria en al menos un diente, el $41.1 \%$ presentó al menos un diente con compromiso de furca y $33.9 \%$ presentó algún diente con caries.

\section{DISCUSIÓN}

Los estudios epidemiológicos utilizan una combinación de conceptos de prevalencia y severidad combinando los conceptos de manera distinta, por lo tanto, es difícil comparar los resultados de los diferentes estudios ${ }^{(9)}$. A su vez, existen pocos estudios epidemiológicos de enfermedad periodontal en América Latina. Específicamente en Chile hay sólo cuatro estudios publicados, los cuales abarcan muestras locales y distintos grupos etarios, utilizando cada uno parámetros propios o diferentes clasificaciones, por lo que dificulta una comparación más cuantitativa. Sin embargo, estos estudios, si bien no permiten generalizar los resultados obtenidos, permiten explorar el estado de salud de un grupo particular, estudiando sujetos con características particulares o aquellos que por consideraciones prácticas están accesibles para el investigador ${ }^{(10)}$, como es en el caso de esta investigación, que se llevó a cabo en la población de adultos de 60 años pertenecientes a los tres consultorios municipalizados de Villa Alemana, utilizando una metodología estándar con los parámetros e índices periodontales más ocupados en estudios internacionales y recomendados por la OMS.

Dado los múltiples fenotipos y expresiones de la enfermedad periodontal es difícil trabajar con diagnóstico, debiendo utilizar parámetros clínicos objetivos que permitan evaluar el estado periodontal. Dentro de los conceptos para describir el estado periodontal, el más usado es el nivel de inserción clínica ${ }^{(11)}$, dado que es el más confiable, mostrando ineludiblemente la experiencia de enfermedad periodontal y el daño acumulado por ésta ${ }^{(10)}$. Asimismo, es el mejor indicador para determinar la gravedad de la enfermedad periodontal y no la profundidad de sondaje, ya que el margen gingival no es un punto fijo desde el cual se pueda medir por sus fluctuaciones hacia nivel apical o coronal, por este motivo, no permite valorar los cambios longitudinales de inserción clínica. No obstante, la profundidad de sondaje es de gran importancia, puesto que proporciona una valorización útil de la localización del hábitat de las bacterias subgingivales, es decir, los sacos periodontales ${ }^{(12)}$.

EI CPITN es el segundo índice más utilizado en estudios epidemiológicos, siendo recomendado por la OMS. Sin embargo, no es un buen parámetro, ya que al ser un registro parcial -utiliza dientes índices- puede subestimar la extensión y gravedad de la enfermedad(13), no describe las características de la enfermedad, no considera la movilidad dentaria ni la perdida de inserción, sobreestima las necesidades de tratamiento y subestima la magnitud y severidad de la enfermedad al ser un registro parcial, es decir, no determina la extensión ni la severidad de la enfermedad periodontal ${ }^{(14)}$. En cambio, el PSR al evaluar todos los dientes es un índice mejor que el CPITN. Sin embargo, presenta las mismas desventajas que el CPITN, puesto que no considera la multiplicidad de sitios examinados ${ }^{(15)}$

Esta investigación, a pesar de lo dicho anteriormente, utilizó estos parámetros periodontales (nivel de inserción clínica, PSR y profundidad de sondaje), porque son los más usados en estudios epidemiológicos, lo que permite hasta cierto punto la comparación de los resultados. Al existir muy pocos estudios enfocados sólo en adultos de 60 años, se compararon los resultados con estudios en adultos mayores de edades cercanas, como por ejemplo, en el rango de edad de 65 a 74 años.

En este estudio se determinó que el $100 \%$ de los adultos de 60 años presentó al menos un diente con nivel de inserción clínica, concordando con los resultados obtenidos por Lopetegui y cols. ${ }^{(16)}$. Esto indica que todos los adultos mayores han tenido experiencia de daño periodontal y no necesariamente la presencia actual de enfermedad periodontal activa. Según el estudio de National Survey of Oral Health in US employed Adults y Seniors de 1985-1986, el 93\% de los adultos mayores entre 60-64 años presentaron en promedio un nivel de inserción clínica entre 1-2 mm, en contraste con nuestro estudio, en que sólo un $26.61 \%$ de los adultos de 60 años se encontró en ese rango, esta diferencia en los resultados puede deberse a las diferencias socioeconómicas de los grupos estudiados y a los tipos de sistema de salud al que pertenecen. En el estudio realizado en Canadá por Gilbert y Geft se observó que el $24.22 \%$ de los adultos mayores de 65 años presentaron en promedio un nivel de inserción clínica $\geq 5 \mathrm{~mm}^{(17)}$. En Viña del Mar Lopetegui y cols. determinaron que el $22 \%$ de los adultos mayores presentó al menos un diente con nivel de inserción clínica $\geq 5$ $\mathrm{mm}^{(16)}$, resultados más comparables a nuestro estudio, que obtuvo un $30.65 \%$ con adultos con nivel de inserción clínica $\geq 5 \mathrm{~mm}$, dada la similitud de las poblaciones estudiadas y la facilidad técnica para detectar un nivel de inserción clínica $\geq 5 \mathrm{~mm}$.

El $73.39 \%$ de los adultos de 60 años de este estudio presentó al menos un diente con profundidad de sondaje $>4 \mathrm{~mm}$, resultado mayor que el encontrado en el estudio de Irigoyen y cols. en México con un $50.4 \%{ }^{(13)}$. Si lo comparamos con dos estudios de adultos mayores en Estados Unidos en los cuales se encontró un $19.2 \%^{(9)}$ y $22 \%{ }^{(17)}$ respectivamente, las variaciones son aun más evidentes, pudiéndose deber a la diferencia de edades de las poblaciones estudiadas, en las cuales la inclusión de pacientes más jóvenes puede ser la causa, además de las distintas oportunidades de tratamiento que presentan.

Según este estudio, el $82.3 \%$ de los adultos de 60 años presentó una necesidad de tratamiento complejo, correspondiendo al código 3 y 4 del PSR, siendo la necesidad más predominante. En el 
estudio de Skrepcinski y Niendorff en Estados Unidos, el $21.1 \%$ de los adultos mayores entre 65-74 años presentó una necesidad de tratamiento periodontal complejo ${ }^{(17)}$, en México Irigoyen y cols. encontraron que el $50.4 \%$ de los adultos de 60 años necesitaba tratamiento periodontal complejo ${ }^{(13)}$, mientras que Lopetegui y cols. en Viña del Mar determinaron que el $59.3 \%$ necesitaba tratamiento periodontal complejo ${ }^{(16)}$. Las grandes diferencias entre los estudios pueden ser explicadas ya que algunos ocupan CPITN y otros PSR, sumado a la realidad de salud periodontal entre los países. Los adultos que requieran este tipo de tratamientos en la salud pública de nuestro país necesitan ser derivados de los centro de salud de atención primaria a los centros de especialidad dependientes del servicio de salud correspondiente.

A pesar que los adultos de 60 años son dados de alta del programa GES, con un mejor estado periodontal, no significa que esta condición se mantenga a largo plazo, ya que no tienen dentro del programa contemplada la mantención periodontal, donde está comprobado que pacientes que recibían periódicas terapias de mantención bajaron significativamente sus profundidades de sondaje, su pérdida de inserción clínica, y se redujo la pérdida dentaria(18). En el programa GES tampoco se le da el suficiente énfasis a la instrucción y motivación en higiene oral, y al no existir mantenciones, no hay oportunidad de refuerzo en la técnica de higiene, pudiendo esperarse que su condición periodontal empeore con el tiempo. La importancia de este estudio es describir el estado periodontal real de la población y determinar la necesidad de tratamiento de los adultos de 60 años beneficiarios del GES, para así evidenciar el énfasis en el tratamiento periodontal complejo con todas sus etapas y la necesaria derivación al especialista, que según nuestro estudio corresponde al $32.3 \%$, por código 4 . Sin embargo, si sumamos a este porcentaje los códigos asterisco, el $73.38 \%$ de los pacientes deberían ser referidos al periodoncista. Mientras que el $82.3 \%$ de los adultos de 60 años necesita tratamiento periodontal complejo.

En cuanto al número de dientes presentes en los adultos de 60 años, se encontró que en promedio presentaron 16.56 dientes, este promedio fue de 15.5 en mujeres y 17.9 en hombres. Esto concuerda con el estudio de Irigoyen y cols. en México, en donde el promedio de dientes presentes en adultos de 60 años fue de 16.1 dientes, siendo 15.5 en hombres y 16.4 en mujeres ${ }^{(13)}$. Un estudio de Dowsett y cols. en Estados
Unidos indicó que el promedio de dientes en adultos entre 55-64 años fue de 24 dientes $^{(14)}$, mientras que en Canadá, Galan y cols. encontraron que los adultos de 60 años tenían en promedio 8.2 dientes $^{(17)}$. En Kenia, Manji y cols. determinaron que los mayores de 55 años en promedio presentaban 24.1 dientes. En Tanzania, Van Palestein y cols. observaron que los adultos entre 45-60 años presentaron un promedio de dientes de 21.9. En Uganda, Tirwonwe y cols. encontraron que los adultos mayores de 65 años presentaban en promedio 28.8 dientes $^{(19)}$. Las diferencias encontradas entre los estudios pueden deberse al distinto acceso a salud bucal de los distintos países, como también a las diferencias producto de la inclusión de pacientes más jóvenes a la muestra.

\section{CONCLUSIONES}

Los pacientes GES de 60 años presentaron un mal estado periodontal determinado por higiene oral, índice hemorrágico, pérdida de inserción clínica y profundidad de sondaje, mostrando un importante deterioro de la salud periodontal de dicha población. La totalidad de los adultos de 60 años necesita tratamiento periodontal de algún tipo, siendo un $82.3 \%$ el que necesita tratamiento periodontal complejo. El programa GES es la última oportunidad garantizada de tratamiento integral otorgado por el sistema público y privado en Chile, por lo cual el profesional debe diagnosticar correctamente y realizar un adecuado plan de tratamiento, permitiendo una mejoría y mantenimiento en la salud periodontal a largo plazo.

\section{AGRADECIMIENTOS}

Los autores desean agradecer la ayuda otorgada por los consultorios de la corporación municipal de Villa Alemana, en especial a la Dra. Jessica Oesterreich, Dra. María Soledad Cepeda y Dra. Lorena Landaeta por su colaboración desinteresada en esta investigación.

\section{REFERENCIAS BIBLIOGRÁFICAS}

1. San Martín C, Villanueva J. Cambios sistémicos en el paciente adulto mayor. Revista Dental de Chile,2002;93(2):11-13.

2. INE. Enfoque estadístico adulto mayor en Chile. Instituto Nacional de Estadísticas, 2007.

3. Marín PP, Guzmán JM, Araya A. Adultos mayores institucionalizados en Chile: ¿cómo saber cuántos son? Rev Méd Chile,2004;132:832-838.

4. MINSAL. Guía clínica: Salud Oral Integral para el Adulto de 60 años. Santiago, Chile. Ministerio de Salud, 2007.

5. Nunn M. Interpretación de la etiología de la periodontitis: resumen de los factores de riesgo periodontales. Periodontol 2000(Ed Esp),2004; 7:11-23.

6. De Rossi S, Slaughter Y. Oral changes in older patients: a clinician's guide. Quintessence Int,2007;38:773-780.

7. INE. Adultos mayores por regiones, comunas y porcentajes. Instituto Nacional de Estadísticas, 2003.

8. Gamonal J, López N, Aranda W. Periodontal conditions and treatment needs, by CPITN, in the 35-44 and 65-74 year old population in Santiago, Chile. Int Dent J,1998;48(2):96-103.

9. Brown J, Löe H. Prevalence, extent, severity and progression of periodontal disease. Periodontol 2000,1993;2:57-71.

10. Mendoza C, Arteaga O, Gamonal J. Investigación epidemiológica en enfermedades periodontales en América Latina. Rev Chil Period Oseoint, 2006;3(3):7-13.

11. Gjermo P, Rösing C, Susin C, Oppermann R. Periodontal diseases in Central and South America. Periodontol 2000,2002;29:70-78.

12. Armitage G. Diagnóstico y clasificación de las enfermedades periodontales. Periodontol 2000(Ed Esp),2005;9:9-21.

13. Irigoyen $M$, Velázquez $C$, Zepeda $M$, Mejía A. Caries dental y enfermedad periodontal en un grupo de personas de 60 ó más años de edad de la Ciudad de México. ADM,1999;56(2):64-69.

14. Dowsett S, Archila L, Segreto V, Eckert G, Kowolik M. Periodontal diseases status of an indigenous population of Guatemala, Central America. J Clin Periodontol,2001;28:663-671.

15. García D, da Silva C, Oppermann R. Validity of Community Periodontal Index of Treatment Needs (CPITN) for population periodontitis screening. Cad. Saúde Pública,2006;22(2):277-283.

16. Lopetegui M, Pinto C, Vergara L. Estado periodontal de los adultos mayores y su necesidad de tratamiento en los consultorios municipalizados de la ciudad de Viña del Mar. Seminario de Tesis. Escuela de Odontología, Universidad de Valparaíso, Chile, 2004.

17. Albandar J. Periodontal diseases in North America. Periodontol 2000, 2002;29:31-69.

18. AAP. Position paper:Periodontal maintenance. J Periodontol, 2003;74:13951401.

19. Baelum V, Scheutz F. Periodontal diseases in Africa. Periodontol,2000, 2002; 29:79-103. 